\title{
A simplified clinical prediction score of chronic kidney disease: A cross-sectional-survey study
}

\author{
Ammarin Thakkinstian ${ }^{*}$, Atiporn Ingsathit ${ }^{1}$, Amnart Chaiprasert ${ }^{2}$, Sasivimol Rattanasiri ${ }^{1}$, Pornpen Sangthawan ${ }^{3}$, \\ Pongsathorn Gojaseni ${ }^{4}$, Kriwiporn Kiattisunthorn ${ }^{5}$, Leena Ongaiyooth ${ }^{5}$ and Prapaipim Thirakhupt ${ }^{6}$
}

\begin{abstract}
Background: Knowing the risk factors of CKD should be able to identify at risk populations. We thus aimed to develop and validate a simplified clinical prediction score capable of indicating those at risk.

Methods: A community-based cross-sectional survey study was conducted. Ten provinces and 20 districts were stratified-cluster randomly selected across four regions in Thailand and Bangkok. The outcome of interest was chronic kidney disease stage I to $\vee$ versus non-CKD. Logistic regression was applied to assess the risk factors. Scoring was created using odds ratios of significant variables. The ROC curve analysis was used to calibrate the cutoff of the scores. Bootstrap was applied to internally validate the performance of this prediction score.

Results: Three-thousand, four-hundred and fifty-nine subjects were included to derive the prediction scores. Four (i.e., age, diabetes, hypertension, and history of kidney stones) were significantly associated with the CKD. Total scores ranged from 4 to 16 and the score discrimination was $77.0 \%$. The scores of $4-5,6-8,9-11$, and $\geq 12$ correspond to low, intermediate-low, intermediate-high, and high probabilities of CKD with the likelihood ratio positive (LR+) of 1, 2.5 (95\% Cl: 2.2-2.7), 4.9 (95\% Cl: 3.9 - 6.3), and 7.5 (95\% Cl: 5.6 - 10.1), respectively. Internal validity was performed using 200 repetitions of a bootstrap technique. Calibration was assessed and the difference between observed and predicted values was 0.045 . The concordance $C$ statistic of the derivative and validated models were similar, i.e., 0.770 and 0.741 .
\end{abstract}

Conclusions: A simplified clinical prediction score for estimating risk of having CKD was created. The prediction score may be useful in identifying and classifying at riskpatients. However, further external validation is needed to confirm this.

\section{Background}

Chronic kidney disease (CKD) is a precursor to end stage kidney disease (ESKD), which requires major intervention in the form of dialysis or transplant. The prevalence of the ESKD in Thailand from 2002 to 2006 was about 220$286 /$ million population throughout the country [1]. CKD tends to increase according to the increased prevalence of diabetes, hypertension, and economic development within a region. Early identification and targeting of individuals with CKD should be encouraged for the purpose of instituting intervention strategies, such as low-protein dietary changes, close monitoring of blood pressure,

\footnotetext{
* Correspondence: raatk@mahidol.ac.th

'Section for Clinical Epidemiology and Biostatistics, Faculty of Medicine,

Ramathibodi Hospital, Mahidol University, Bangkok, Thailand

Full list of author information is available at the end of the article
}

control of blood sugar levels, health-monitoring programs, education, exercise, and so on[2].

If the risk factors of CKD are known, one should be able to predict the probability of at risk individuals developing CKD, and thus identify at risk populations. Although many previous studies have assessed the risk factors of CKD in general populations, few non-Asian-based studies have constructed prediction scores using cumulative combinations of risk factors [3-6]. A hospital-based study by Hemmelgarn et al[4] studied subjects of ages 66 years or older, and thus applying the score to general population will result in poor validity. Two community-based observational studies $[3,5]$ developed and validated a simple algorithm for CKD stage III or higher based on two demographic data and six medical histories. Among those medical histories, few variables (i.e., a history of heart disease, heart failure, and peripheral vascular disease) were not

\section{() Biomed Central}


easily assessed in a community-base setting, and once they were assessed, their validity was still questionable, particularly in developing countries where education \& knowledge about the diseases are limited. Thus the scores are not qualified as for a concept of developing a simplified prediction score [7-9], in which the scores should not contain many variables and which should be easily and validly measured. Some prediction scores for diabetes had also been used to predict CKD, but discriminative ability was low[6]. We therefore conducted a study to develop and validate a simplified clinical prediction score for estimate risk of developing CKD in the Thai general population. The scores would aid general-practice physicians in identifying individuals who are at risk of having CKD and should have further investigation and management.

\section{Methods \\ Studied population}

This study was part of a community-based, cross-sectional survey study of CKD prevalence where the details of the research methodologies have been clearly described elsewhere [10], so are only briefly described as follows: The study included subjects who were 18 years or older, had no menstruation period for at least a week prior to the examination date if women, and whom were willing participants of the study and provided signed consent forms. Ten provinces and 20 districts were selected across four regions of Thailand (i.e., Northern, Northeastern, Central, Southern) and Bangkok using a stratified-cluster random sampling. Subjects in the sample districts were then randomly selected and stratified by age and sex. The study was approved by two Institutional Review Boards (IRBs), i.e., the IRB of the Faculty of Medicine at Ramathibodi Hospital, Mahidol University, and the IRB of the Ministry of Public Health.

\section{Measurement of risk factors}

Physical examinations (i.e., respiratory rate, weight, height, waist \& hip circumference, and blood pressure) were collected by nurses at each site. Blood samples, after eighthour overnight fasting, were collected for blood chemistry tests (i.e., serum blood sugar, triglycerides (TRIG), total cholesterol (TC), high-density lipoprotein cholesterol (HDL-c), low-density lipoprotein cholesterol (LDL-c), uric acid (UA), serum creatinine, and complete blood count (Hb, WBC). Urine tests (i.e., micro-, macro-protein and urinary analysis) were also collected. Hematuria was defined as the presence of more than 5 red blood cells per high-power field, and microalbuminuria was defined as an albumin-creatinine ratio of 30 to $300 \mathrm{mg} / \mathrm{g}$. Hypertension, diabetes, and high cholesterol, were classified based on one of following conditions: a history of illnesses, relevant medicines used (e.g., non-steroidal anti-inflammatory drug (NSAID)), or laboratory tests/physical examinations.
Anemia was diagnosed if subjects had hemoglobin levels of less than $10 \mathrm{~g} / \mathrm{dl}$. History of kidney stone was measured by self-reporting kidney stone.

\section{Outcome measurements}

Serum creatinine was measured using the modified Kinetic Jaffe (KJ) method and then standardized by the isotope dilution mass spectrometry (IDMS) method. The estimated glomerular filtration rate (GFR) was then calculated using an MDRD equation for IDMS traceable serum creatinine values as follows[11]: eGFR $\left(\mathrm{mL} / \mathrm{min} / 1.73 \mathrm{~m}^{2}\right)=$ $175 \times(\mathrm{SCr})^{-1.154} \times(\text { Age })^{-0.203} \times(0.742$ if female $)$.

CKD was defined [12] as stage I \& II if GFR $\geq 90$ and GFR $60-89 \mathrm{ml} / \mathrm{min} / 1.73 \mathrm{~m}^{2}$ with haematuria and/or albumin-creatinine ratio $30 \mathrm{mg} / \mathrm{g}$ or greater, stage III, IV, and $\mathrm{V}$ if the GFR of $30-59,15-29$, and $<15 \mathrm{ml} / \mathrm{min} / 1.73 \mathrm{~m}^{2}$ respectively, regardless of kidney damage. The CKD stages $\mathrm{I}-\mathrm{V}$ were combined and then compared with non-CKD in analysis.

\section{Statistical analysis \\ Derivative phase}

Data from the 10 provinces were used to derive a simplified clinical prediction score. Weighted logistic regression for a multi-stage sampling survey data was applied to derive the parsimonious model. The three-stage weight was calculated briefly as follows: 1 /[probability of sampling provinces $] \times[$ probability of sampling districts $] \times[$ probability of sampling subjects]]. The probability of sampling provinces was estimated by the number of sample provinces divided by the total number of provinces in that stratum (region). The probability of sampling districts was calculated by the number of sample districts divided by the total number of districts in the sample province. Finally, the probability of sampling subjects was the number of subjects divided by the size of the population of the sample district. Data from the Thai population census of 2007, Ministry of Interior [13] was used for population size.

Factors with $\mathrm{p}$ values $<0.15$ in a univariate analysis were considered to be simultaneously included in the multivariate logistic equation. Model selection was performed using F-tests, and thus only significant variables were kept in the final model. Receiver operating characteristic (ROC) analysis was applied to simplify the model and the area under the ROC curve or known as the concordance $(C)$ statistic was estimated. The $C$ statistic of models with and without a particular variable were then compared; if dropping that variable did not significantly reduce the explanation of the $\mathrm{CKD}$, that variable was omitted in the final parsimonious model. Model's performances of the final model measured by the goodness of fit statistic and the $\mathrm{C}$ statistic were assessed. The odds ratios (OR) of the final model were then estimated and rounded off in order to simplify the scores, and these were used to construct the scoring 
scheme. Individuals were allocated scores relevant to each variable and summed up as total scores. Score performances, i.e., sensitivity, specificity, and positive and negative likelihood ratios $\left(\mathrm{LR}^{+} / \mathrm{LR}^{-}\right)$were calculated according to each possible total score. The total scores were then classified according to the strength of the $\mathrm{LR}^{+}$[14]. Post test probability was next estimated.

\section{Validation phase}

Internal validation of the prediction scores was checked using a bootstrap of 200 repetitions [15,16]. For each sample of the bootstrap, the logistic model was fitted based on significant variables in the derivative phase, and prediction scores based on ORs and parameters (i.e. predicted probability and the $C$ statistic) were estimated. The correlation between the observed and predicted values of CKD was assessed using the Somer'D correlation, called $\mathrm{D}_{\text {boot }}$. Calibration of the model was then assessed by subtracting the original Somer'D correlation from the mean $\mathrm{D}_{\text {boot }}$. Discrimination of the model was assessed by comparing the original $C$ statistic versus an average $C$ statistic from the bootstraps. All analyses were performed using STATA 11. A p value $<0.05$ was considered statistically significant.

\section{Results}

\section{Derivative phase}

All 3,459 subjects from the CKD prevalence study[10] were included to derive the prediction scores. The characteristics of the subjects were described (table 1), i.e., the mean age was 45.2 years $(\mathrm{SE}=0.79), 54.5 \%$ were females and $63.9 \%$ had BMI less than $25 \mathrm{~kg} / \mathrm{m}^{2}$. The mean waisthip ratio was $0.81(\mathrm{SE}=0.01)$. The majority of subjects had never smoked (64.1\%), and some of them were currently or ever alcohol drinkers (58.9\%). The prevalence of diabetes, hypertension, and high cholesterol were frequent, i.e., $11.9 \%, 27.5 \%$, and $26.4 \%$, respectively. However, a history of heart disease, cerebrovascular accident, and history of kidney stones were quite low, i.e., 3.4\%, 1.4\%, and 5.0\% respectively. Interestingly, history of taking NSAID, and traditional medicines were as high as $44.7 \%$ and $33.5 \%$, respectively. Mean serum creatinine in males and females were $1.1(\mathrm{SE}=0.02)$ and $0.8(\mathrm{SE}=0.02)$ respectively. The CKD prevalence was $17.5 \%$ (95\% confidence interval (CI): $14.6 \%-20.5 \%)$.

Sixteen variables were considered in the univariate analysis, as shown in Table 1. All variables (except gender, smoking, exercise, LDL, and NSAIDs) with $\mathrm{p}$ values $<0.15$ were simultaneously included in a multivariate model. The final model contained only 4 variables (i.e., age, diabetes, hypertension, and history of kidney stones), and their ORs are listed in Table 2. The scoring scheme for each variable was constructed by rounding off the ORs and assigning 1 to the reference category. Point scores of 8,4 , and 2 were assigned for ages $>70,60-69,40-59$ years; 3 points for each of diabetes and history of kidney stone, and 2 points for hypertension. Total scores ranged from 4 to16 and the score discriminative ability was $77.0 \%$, as shown in Figure 1. The goodness of fit was assessed and found the final model was fit well with our data ( $F$ test $=3.02, p=$ 0.264). For ease of use and simplicity, the range of scores was classified into 4 groups according to $\mathrm{LR}^{+}$; scores of 4$5,6-8,9-11$, and $\geq 12$ correspond to low, intermediatelow, intermediate-high, and high probabilities of having CKD with the $\mathrm{LR}^{+}$of 1, 2.5 (95\% CI: 2.2-2.7), 4.9 (95\% CI: 3.9 - 6.3), and 7.5 (95\% CI: 5.6 - 10.1), respectively. The post-test probability was estimated using the prevalence of CKD as $17.5 \%$ (95\% CI: $14.6 \%-20.2 \%$ ) according to findings from our previous study [10], as detailed in table 3. An illustrative example of using the scheme is as follows: A patient aged 55 years, with history of diabetes and hypertension but no history of kidney stone, would be scored as $2+3+2+0=7$; this is categorized as an intermediate-low risk of having CKD. Assuming a baseline prevalence (pre-test probability) of CKD of 14.6 to $20.4 \%$, the post-test probability is $\sim 29 \%$ to $40 \%$.

\section{Validation phase}

Two-hundred repetitions of bootstrap were performed and the logit model contained 4 variables shown in table 2 which was constructed for each bootstrap. The estimated Somer'D correlation coefficeints for the original and the bootstrap models were 0.544 and 0.499, respectively. Calibration of the model was assessed by subtracting the two Somer's D correlation coefficients, and it was found that the bias, according to a difference in observed versus predicted values, was only 0.045 (95\% CI:0.034, 0.057). The C statistic of the derivative and validated models were very much similar, i.e., 0.770 and 0.741 . The average difference (known as degree of optimism) was 0.029 . This suggested that the score can fairly accurately discriminate CKD from non-CKD if it is applied to a general population whose life styles are similar to the Thai general population.

\section{Discussion}

We have developed and validated a simplified clinical prediction score for classifying populations into mild, intermediate-low, intermediate-high, and high risks of developing CKD. The score was made easier and simplified using factors that were readily available and simply assessed in clinical practice. The score shows good calibration and discrimination, as it can be seen from a similarity between observed and predicted values, as well as between the $\mathrm{C}$ statistic in the derivative and validation phases, respectively.

We mainly focus on estimating risk of developing CKD, not CKD progression. Our study has strengths in research methods as commented in detail in Ingsathit et al[10]. It is derived from a large number of subjects who were 
Table 1 Patient characteristics between CKD and Non-CKD groups

\begin{tabular}{|c|c|c|c|c|c|c|c|c|}
\hline \multirow[t]{3}{*}{ Factors } & & \multirow[t]{3}{*}{ Total $(\% *)$} & \multicolumn{4}{|c|}{ CKD } & \multirow[t]{3}{*}{ OR $(95 \% \mathrm{Cl})$} & \multirow[t]{3}{*}{ p-value } \\
\hline & & & \multicolumn{2}{|c|}{ Stage I-V } & \multicolumn{2}{|c|}{ Normal } & & \\
\hline & & & number & $\%$ & Number & $\%$ & & \\
\hline Age & & $45.2(0.79) * *$ & & & & & & \\
\hline$\geq 70$ & & $267(7.7)$ & 139 & 22.3 & 128 & 4.1 & $14.8(8.5,26.0)$ & $<0.001$ \\
\hline $60-69$ & & $403(11.8)$ & 148 & 22.9 & 255 & 9.4 & $6.6(4.2,10.3)$ & $<0.001$ \\
\hline $40-59$ & & $1464(43.0)$ & 237 & 39.2 & 1,227 & 43.8 & $2.4(1.8,3.3)$ & 0.001 \\
\hline$<40$ & & 1325 (37.9) & 102 & 15.7 & 1,223 & 42.7 & 1 & \\
\hline \multicolumn{9}{|l|}{ Gender } \\
\hline Male & & 1569 (45.5) & 356 & 57.8 & 1,534 & 53.9 & $1.2(0.9,1.6)$ & 0.253 \\
\hline Female & & $1890(54.5)$ & 270 & 42.2 & 1,299 & 46.1 & 1 & \\
\hline BMI & & $24(0.2)^{* *}$ & & & & & & \\
\hline$\geq 30$ & & $285(8.9)$ & 65 & 11.7 & 220 & 8.3 & $1.6(1.2,2.2)$ & 0.014 \\
\hline $25-29.9$ & & $924(27.3)$ & 191 & 30.5 & 733 & 26.6 & $1.3(1.0,1.7)$ & 0.045 \\
\hline$<25$ & & $2250(63.9)$ & 370 & 57.8 & 1,880 & 65.1 & 1 & \\
\hline Waist/Hip & & $0.84(0.01) * *$ & & & & & & \\
\hline Male: & Female: & & & & & & & \\
\hline$\geq 0.96$ & $\geq 0.90$ & $354(9.3)$ & 117 & 18.6 & 237 & 7.4 & $2.9(1.7,4.8)$ & 0.004 \\
\hline$<0.96$ & $<0.90$ & 3104 (90.7) & 509 & 81.4 & 2,595 & 92.6 & 1 & \\
\hline \multicolumn{9}{|l|}{ Smoking } \\
\hline Yes & & 1251 (35.9) & 232 & 36.4 & 1,019 & 35.8 & $1.0(0.7,1.5)$ & 0.864 \\
\hline No & & $2194(64.1)$ & 391 & 63.6 & 1,803 & 64.2 & 1 & \\
\hline \multicolumn{9}{|c|}{ Alcohol consumption } \\
\hline Yes & & $2084(59.0)$ & 326 & 49.5 & 1,758 & 61.0 & $1.6(1.0,2.5)$ & 0.044 \\
\hline No & & $1360(41.0)$ & 299 & 50.5 & 1,061 & 39.0 & 1 & \\
\hline \multicolumn{9}{|l|}{ Exercise } \\
\hline Yes & & $2057(59.8)$ & 380 & 61.9 & 1,677 & 59.4 & $1.1(0.9,1.3)$ & 0.164 \\
\hline No & & $1390(40.1)$ & 242 & 38.1 & 1,148 & 40.6 & 1 & \\
\hline \multicolumn{9}{|c|}{ Work involving significant activity } \\
\hline Yes & & $2,115(57.9)$ & 323 & 49.2 & 1,792 & 59.9 & $1.5(1.1,2.1)$ & 0.016 \\
\hline No & & $1,296(42.0)$ & 297 & 50.8 & 999 & 40.1 & 1 & \\
\hline \multicolumn{9}{|l|}{ DM } \\
\hline Yes & & $434(11.9)$ & 183 & 28.5 & 251 & 8.4 & $4.3(2.9,6.6)$ & $<0.001$ \\
\hline No & & $3,025(88.1)$ & 443 & 71.5 & 2,582 & 91.6 & 1 & \\
\hline \multicolumn{9}{|c|}{ Hypertension } \\
\hline Yes & & 955 (27.5) & 329 & 53.6 & 626 & 22.0 & $4.1(2.9,5.7)$ & $<0.001$ \\
\hline No & & $2,504(72.5)$ & 297 & 46.4 & 2,207 & 78.0 & 1 & \\
\hline \multicolumn{9}{|c|}{ High cholesterol } \\
\hline Yes & & $851(26.4)$ & 203 & 34.3 & 648 & 24.7 & $1.6(1.2,2.1)$ & 0.007 \\
\hline No & & 2,608 (73.6) & 423 & 65.7 & 2,185 & 75.3 & 1 & \\
\hline \multicolumn{9}{|c|}{ Kidney stone } \\
\hline Yes & & $169(5.0)$ & 74 & 11.3 & 95 & 3.7 & $3.3(2.1,5.2)$ & 0.001 \\
\hline No & & $3,085(95.0)$ & 516 & 88.7 & 2,569 & 96.3 & 1 & \\
\hline \multicolumn{9}{|l|}{ LDL } \\
\hline$\geq 160$ & & 591 (19.2) & 116 & 20.9 & 475 & 18.8 & $1.1(0.8,1.6)$ & 0.490 \\
\hline 130-159 & & 807 923.3) & 134 & 21.4 & 673 & 23.7 & $0.9(0.7,1.1)$ & 0.263 \\
\hline$<130$ & & 1979 (57.5) & 359 & 57.7 & 1,620 & 57.5 & 1 & \\
\hline \multicolumn{9}{|l|}{ Uric acid } \\
\hline$>5.61$ & & 1,269 (38.6) & 331 & 55.0 & 938 & 35.1 & $2.7(1.8,4.0)$ & 0.001 \\
\hline $4.40-5.61$ & & $1,126(32.3)$ & 166 & 26.6 & 960 & 33.5 & $1.4(0.9,2.1)$ & 0.123 \\
\hline$<4.40$ & & $1,064(29.1)$ & 129 & 18.4 & 935 & 31.4 & 1 & \\
\hline
\end{tabular}


Table 1 Patient characteristics between CKD and Non-CKD groups (Continued)

\begin{tabular}{lccccccc}
\hline Yes & $1,143(33.5)$ & 263 & 42.6 & 880 & 31.5 & $1.6(1.4,1.9)$ & 0.001 \\
No & $2,300(66.5)$ & 361 & 57.4 & 1,939 & 68.5 & 1 & \\
NSAIDs & & & & & & \\
Yes & $1,577(44.7)$ & 308 & 48.3 & 1,269 & 43.9 & $1.2(0.8,1.7)$ & 0.266 \\
No & $1,882(55.3)$ & 318 & 51.7 & 1,564 & 56.1 & 1 & \\
\hline
\end{tabular}

${ }^{*}$ A sampling weight was applied to calculate percentage, ${ }^{* *}$ mean (SE)

stratified-cluster randomly sampling form areas across Thailand. In addition, we have validated the clinical prediction score using a 200-repetition bootstrap technique, which is considered a good technique for internal validation [15-17]. The $\mathrm{C}$ statistic of the simplified score was fair in both derivative and bootstrap data (i.e., 0.77 and 0.74 ), indicating that the score can well discriminate between CKD and non-CKD subjects. Our model is simplified and should be easily to apply in clinical practice because of required variables are routinely measured. The scoring scheme should be able to apply manually by clinicians and then the score is classified into 4 groups without requiring any calculation.

We however have some limitations. Our design was a cross-sectional survey study and thus the temporal sequence between risk factors and CKD was questionable[18]. External validation has not been performed and generalizability of our prediction model is still needed to determine. Given good represent samples across the country, the model might work well in outside the studied areas in Thailand, or in other countries where prevalence of diabetes $(\sim 11.9 \%)$, hypertension (27.5\%), and kidney stone $(5.0 \%)$ are similar to Thai population. The overly-optimistic predictions in other populations might be less likely because of an optimistic rate from the bootstrap is only $2.9 \%$.
To our knowledge, only a few previous prediction scores $[3-5,19]$ for kidney diseases were available in prior literature. The study by Hemmelgarn et al [4] had developed a clinical index for rapid progression of kidney function, which was defined as a decline in eGFR of $25 \%$ or greater. Five predictors were included in the clinical index, which were age, heart disease, diabetes, gout, and the use of anti-emetic drugs. Comparatively, in our study, only two predictors (i.e., age and diabetes) were similar, but the others were not significantly associated with CKD and thus were not considered. The ability of the score to correctly discriminate those individuals with and without CKD from our study was fair (area under ROC = 0.77 ) but it was low for this study (area under ROC = 0.59). Bang et al[3] had well developed scores based on a cross-sectional NHANES 1999-2000 \& 2001-2002 data. The performance of their scores had also been both internally and externally tested with good and fair performances (i.e., the $C$ statistic were 0.88 and 0.71 , respectively). A suggestion of using the score in clinical practice was also discussed. However, applying a score with a required 9 input variables (i.e., age, female, anemia, hypertension, diabetes, history of cardiovascular disease, congestive heart failure, peripheral vascular disease, and proteinuria) in clinical practice might not be as simple as suggested. Data for a history of cardiovascular disease,

Table 2 Factors associated with CKD and creating a scoring scheme: Multiple logistic regression analysis

\begin{tabular}{|c|c|c|c|c|c|c|}
\hline Factors & Coefficient & SE & P-value & OR $(95 \% \mathrm{Cl})$ & Scoring & Score for individual \\
\hline \multicolumn{7}{|l|}{ Age } \\
\hline$\geq 70$ & 2.1 & 0.22 & $<0.001$ & $8.3(4.7,14.4)$ & 8 & \\
\hline $60-69$ & 1.4 & 0.17 & $<0.001$ & $4.1(2.6,6.3)$ & 4 & \\
\hline $40-59$ & 0.6 & 0.13 & 0.007 & $1.8(1.3,2.5)$ & 2 & \\
\hline$<40$ & & & & 1 & 1 & $\ldots \ldots \ldots \ldots \ldots \ldots$ \\
\hline \multicolumn{7}{|l|}{ Kidney stone } \\
\hline Yes & 1.0 & 0.15 & 0.001 & $2.8(1.9,4.1)$ & 3 & \\
\hline No & & & & 1 & 1 & $\ldots \ldots \ldots \ldots \ldots \ldots$ \\
\hline \multicolumn{7}{|l|}{ DM } \\
\hline Yes & 0.9 & 0.19 & 0.005 & $2.5(1.5,4.1)$ & 3 & \\
\hline No & & & & 1 & 1 & $\ldots \ldots \ldots \ldots \ldots \ldots \ldots \ldots$ \\
\hline \multicolumn{7}{|l|}{ Hypertension } \\
\hline Yes & 0.8 & 0.13 & 0.002 & $2.3(1.6,3.2)$ & 2 & \\
\hline No & & & & 1 & 1 & $\ldots \ldots \ldots \ldots \ldots \ldots \ldots \ldots$ \\
\hline \multicolumn{5}{|c|}{ Total score } & $4-16$ & 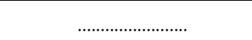 \\
\hline
\end{tabular}




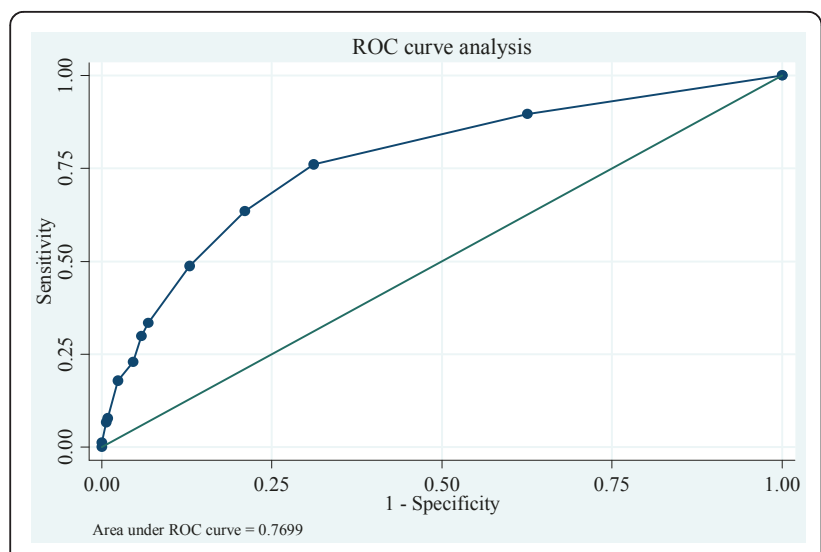

Figure 1 Performance of a clinical prediction score of CKD: ROC curve analysis.

congestive heart failure, and peripheral vascular disease may not be valid in developing countries where populations have a limited understanding of the diseases they have been diagnosed with. The ability of discrimination for this score in Asians may not be valid since the prevalence of CKD in Caucasian vs. Asian populations is quite different [20], and also lifestyle factors that directly or indirectly influence CKD may be markedly different. Kshirsagar et al[5] conducted a study on a communitybased cohorts with ages of 45 years or older in order to create the best fitting and simplified scores for predicting the incidence of CKD (GFR $<60 \mathrm{ml} / \mathrm{min} / 1.73 \mathrm{~m}^{2}$ ). The study design was better than previous studies in terms of the predictor-CKD causal relationship, which could be assessed for the cohort study. Two prediction score models were proposed, one with, the full score of 10 predictors (i.e., age, white ethnicity, female, anemia, hypertension, diabetes, history of cardiovascular disease, history of heart failure, low HDL, and peripheral vascular disease), and a simplified score with 8 predictors, omitting two variables, ethnicity and HDL. Again, applying either the best fitting or the reduced model may not so simple since so many variables are required to input in the models. Creating a score by rounding up a coefficient to the nearest integer (higher than estimated) was not clearly described in the paper. For instance, estimated coefficients for ages $60-69$ and $\geq 70$ years of 1.31 and 1.46 respectively were rounded up to be 2 and 3 instead of rounding down to 2 and 2. In addition, coefficients of other variables that were less than 1 (e.g., 0.2-0.6) were rounded up to be equal to a score of 1 and thus given an equal weight of contribution to the total scores. This would raise the question, for example, of whether gender played the same role as hypertension. The clinical prediction scores for diabetes have also been used for predicting $C K D[6]$. As expected, the discrimination was low, ranging from 0.60 to 0.71 , and generalizability was questionable.

\section{Using the clinical prediction score in practice}

Using only one cut-off (e.g., according to Yuden's index (sensitivity + specificity - 1)[21,22]) classifies subjects too broadly and thus does not work for this prediction score. For instance, applying Yuden's index resulted in a cut-off of 5 which provided the highest sensitivity and specificity (i.e., $76 \%$ and $69 \%$, respectively). This would also result in a very large screening of serum creatinine across the country if a suggestion were based on this cutoff. In a country with limited resources, scoring should be prioritized with meaningful and clinical relevance. The prediction scores are thus classified into four groups according to the $\mathrm{LR}^{+}$which are: low (4-5); intermediate-low (6-8); intermediate-high (9-11); and high $(\geq 12)$. The variables used are easily obtained and measurable in general practice; hence, a general practitioner, an internist, or even a nephrologist should be able to manually apply prediction scores in routine bedside-practice. Interpreting $\mathrm{LR}^{+}$is more informative using a nomogram, which is widely used in diagnostic test results [23]. For instance, in data from a medical record or physical examination, if a subject is younger than 40 years with high blood pressure, no history of diabetes and history of kidney stones, and normal blood sugar, this would give the patient a score of 5 , corresponding to a $\mathrm{LR}^{+}$of 1 . Assuming a baseline prevalence (pre-test probability) of CKD ranging from 17.5\% (95\% CI: $14.6 \%-20.2 \%$ )[10], the post-test probability of this person is $\sim 17.5 \%$ (95\% CI: $15 \%-20 \%$ ). This patient is in a low risk group and thus can be checked for kidney function once a year, if and only if he/she does not develop other risks, e.g., diabetes or history of kidney stones. If the patient later develops diabetes and/or kidney stones, this would give him/her a score of 6-8, corresponding to a $\mathrm{LR}^{+}$of 2.5 and the post-test odds would be

Table 3 Predictive values of a clinical prediction score

\begin{tabular}{ccccc}
\hline Score & Sensitivity (\%) & Specificity (\%) & +LR (95\% Cl) & Post-test probability (95\% CI) $^{*}$ \\
\hline $4-5$ & 100.0 & 0 & 1 & $17.5 \%(15 \%-20 \%)$ \\
$6-8$ & 76.1 & 68.8 & $2.5(2.2-2.7)$ & $35 \%(29 \%-40 \%)$. \\
$9-11$ & 33.4 & 93.2 & $4.9(3.9-6.3)$ & $50 \%(45 \%-55 \%)$ \\
$\geq 12$ & 17.9 & 97.6 & $7.5(.6-10.1)$ & $60 \%(55 \%-65 \%)$ \\
\hline
\end{tabular}

*Based on CKD prevalence of $17.5 \%$ and its $95 \%$ Cl of $14.6 \%-20.4 \%$ 
0.530 (i.e., a pre-test odds $\left.\times \mathrm{LR}^{+}=0.175 /(1-0.175) \times 2.5\right)$. This resulted in the post-test probability of $\sim 35 \%$ (95\% CI: $29 \%-40 \%$, which could be estimated by post-test odds/(1+post-test odds). Monitoring kidney function by measuring both serum creatinine and urine albumin should be more frequent than a score of 5 , say twice a year. Subject ages $<40$ to 59 years having all 3 risks would have scores of 9-11, giving a $\mathrm{LR}^{+}$of 4.9 which would result in a post-test probability of $\sim 50 \%$ (95\% CI: $45 \%-55 \%$ ). If he/she is getting older, say age $\geq 60$ years, the score is 12 with $\mathrm{LR}^{+}$of $\sim 7$, resulting in a post-test probability of $\sim 60 \%$ (95\% CI: $55 \%-65 \%$ ). These two scores give intermediate-high and high probabilities of having CKD, and thus indicating more frequent follow-ups, say 3-4 times a year. Treatments for diabetes and hypertension should be also intensified according to guideline of treatments to control disease conditions. As a result, risk of developing CKD is lowering or once it occurs, delay progression of CKD will be targeted.

\section{Conclusion}

We propose a simple clinical prediction score of CKD to aid general practitioners, internists, or nephrologists in identifying CKD in the general population. Practioners should be encouraged to use the score in routine clinical practice in order to make a more concerted effort in the identification and early treatment of CKD. Vigilant monitoring should be planned to prevent the development of CKD and delay higher stages if it happens. In the long run, this prediction score will be of benefit to the country if end-stage kidney disease can be reduced.

\section{List of abbreviations}

AUC: Area under curve; CKD: Chronic kidney disease; Cl: Confidence interval; ESKD: end stage kidney disease; HDL-C: High-density lipoprotein cholesterol; IDMS: Isotope dilution mass spectrometry; KJ: modified Kinetic Jaffe; LDL-C: Low-density lipoprotein cholesterol; +LR/-LR: Positive and negative likelihood ratios; NSAID: Non-steroidal anti-inflammatory drug; OR: Odds ratio; ROC: Receiver operating characteristic; TRIG: triglycerides; TC: total cholesterol; UA: Uric acid

\footnotetext{
Acknowledgements

Thai-SEEK group:

Members of Thai-SEEK group below had contributed in data collection, performing laboratory tests, or general management at the camp sites. Ajay K. Singh, M.D., Renal Division, Brigham and Women's Hospital and Harvard Medical School, Boston, USA

Anchalee Chitthamma, Ph.D., Division of Clinical Chemistry, Department of Pathology, Faculty of Medicine, Ramathibodi Hospital

Bharati Mittal, Renal Division, Brigham and Women's Hospital and Harvard Medical School, Boston, USA

Chaiyot Warunyuwong, MD., Department of medicine, Chon Buri Hospital, Cholburi, Thailand

Dhavee Sirivongs, M.D., Division of Nephrology, Department of Medicine, Faculty of Medicine, Khon Kaen University, Khon Kaen, Thailand

Osot Nerapusee, Pharmacist, Medical Affairs Director, Thai Janssen-Cilag Ltd. Somlak Vanavanan, M.Sc., Division of Clinical Chemistry, Department of Pathology, Faculty of Medicine, Ramathibodi Hospital

Talerngsak Kajanabuch, MD, Faculty of Medicine, Chulalongkorn University
}

Porntip Chatchaipan, M.Sc., Section for Clinical Epidemiology and Biostatistics, Faculty of Medicine, Ramathibodi Hospital

Thananda Trakarnvanich, MD, Renal Unit, Department of Medicine, Bangkok Metropolitan Administration Medical College and Vajira Hospital Vuddhidej Ophascharoensuk, MD, Faculty of Medicine, Chiang-Mai University Hospital

Warangkana Pichaiwong, MD, Division of Nephrology, Department of Medicine, Faculty of Medicine, Rajvithee Hospital, Bangkok, Thailand The Nephrology Society of Thailand and Thai Janssen-Cilag Ltd. This study was partly granted by Thai Janssen-Cilag Ltd and National Health Security Office of Thailand.

\section{Author details}

'Section for Clinical Epidemiology and Biostatistics, Faculty of Medicine, Ramathibodi Hospital, Mahidol University, Bangkok, Thailand. ²Division of Nephrology, Department of Medicine, Phramongkutklao Hospital, Bangkok, Thailand. ${ }^{3}$ Division of Nephrology, Department of Medicine, Faculty of Medicine, Prince of Songkla University, Songkla, Thailand. ${ }^{4}$ Division of Nephrology, Department of Medicine, Bhumibol Adulyadej Hospital, Bangkok, Thailand. ${ }^{5}$ Division of Nephrology, Department of Medicine, Faculty of Medicine, Siriraj Medical School and Hospital, Mahidol University, Bangkok, Thailand. ${ }^{6}$ Division of Nephrology, Department of Pediatrics,

Phramongkutklao Hospital, Bangkok, Thailand.

\section{Authors' contributions}

AT, Al, AC contributed to concept and study design. AT, Al, AC, PS, PG, KK, $\mathrm{LO}$, and PT had involved in acquisition of data. AT and SR performed data analysis, interpretation of results, and drafting the manuscript. AT, Al, AC, LO and PT critically revised the manuscript. All authors read and approved the final manuscript.

\section{Competing interests}

Al received travel fund from Pfizer. AT received honorarium from this project. The other authors declare that they have no competing interest.

Received: 20 January 2011 Accepted: 26 September 2011

Published: 26 September 2011

\section{References}

1. International comaparisons of ESRD. [http://www.usrds.org/2008/pdf/ V2_12_2008.pdf].

2. McClellan WM: Epidemiology and risk factors for chronic kidney disease. The Medical clinics of North America 2005, 89(3):419-445.

3. Bang H, Vupputuri S, Shoham DA, Klemmer PJ, Falk RJ, Mazumdar M, Gipson D, Colindres RE, Kshirsagar AV: SCreening for Occult REnal Disease (SCORED): a simple prediction model for chronic kidney disease. Archives of internal medicine 2007, 167(4):374-381.

4. Hemmelgarn BR, Culleton BF, Ghali WA: Derivation and validation of a clinical index for prediction of rapid progression of kidney dysfunction. Qjm 2007, 100(2):87-92.

5. Kshirsagar AV, Bang H, Bomback AS, Vupputuri S, Shoham DA, Kern LM, Klemmer PJ, Mazumdar M, August PA: A simple algorithm to predict incident kidney disease. Archives of internal medicine 2008, 168(22):2466-2473

6. Lin JW, Chang YC, Li HY, Chien YF, Wu MY, Tsai RY, Hsieh YC, Chen YJ, Hwang JJ, Chuang LM: Cross-sectional validation of diabetes risk scores for predicting diabetes, metabolic syndrome, and chronic kidney disease in Taiwanese. Diabetes care 2009, 32(12):2294-2296.

7. Kuijer PM, Hutten BA, Prins MH, Buller HR: Prediction of the risk of bleeding during anticoagulant treatment for venous thromboembolism. Archives of internal medicine 1999, 159(5):457-460.

8. van Houwelingen HC: Validation, calibration, revision and combination of prognostic survival models. Statistics in medicine 2000, 19(24):3401-3415.

9. Wilson PW, D'Agostino RB, Levy D, Belanger AM, Silbershatz H, Kannel WB: Prediction of coronary heart disease using risk factor categories. Circulation 1998, 97(18):1837-1847.

10. Ingsathit A, Thakkinstian A, Chaiprasert A, Sangthawan P, Gojaseni P, Kiattisunthorn K, Ongaiyooth L, Vanavanan S, Sirivongs D, Thirakhupt $P$, et al: Prevalence and risk factors of chronic kidney disease in the Thai adult population: Thai SEEK study. Nephrol Dial Transplant 2010. 
11. Coresh J, Selvin E, Stevens LA, Manzi J, Kusek JW, Eggers P, Van Lente F, Levey AS: Prevalence of chronic kidney disease in the United States. Jama 2007, 298(17):2038-2047.

12. Levey AS, Eckardt KU, Tsukamoto Y, Levin A, Coresh J, Rossert J, De Zeeuw D, Hostetter TH, Lameire N, Eknoyan G: Definition and classification of chronic kidney disease: a position statement from Kidney Disease: Improving Global Outcomes (KDIGO). Kidney Int 2005, 67(6):2089-2100.

13. Department of provincial administration, Ministry of Interior. [http:// www.dopa.go.th/]

14. Grimes DA, Schulz KF: Refining clinical diagnosis with likelihood ratios. Lancet 2005, 365(9469):1500-1505.

15. Harrell FE Jr, Lee KL, Mark DB: Multivariable prognostic models: issues in developing models, evaluating assumptions and adequacy, and measuring and reducing errors. Statistics in medicine 1996, 15(4):361-387.

16. Schumacher M, Hollander N, Sauerbrei W: Resampling and cross-validation techniques: a tool to reduce bias caused by model building? Statistics in medicine 1997, 16(24):2813-2827.

17. Altman $\mathrm{DG}$, Royston $\mathrm{P}$ : What do we mean by validating a prognostic model? Statistics in medicine 2000, 19(4):453-473.

18. Grimes DA, Schulz KF: Cohort studies: marching towards outcomes. Lancet 2002, 359(9303):341-345

19. Bang H, Mazumdar M, Kern LM, Shoham DA, August PA, Kshirsagar AV: Validation and comparison of a novel screening guideline for kidney disease: KEEPing SCORED. Archives of internal medicine 2008, 168(4):432-435.

20. Anothaisintawee T, Rattanasiri S, Ingsathit A, Attia J, Thakkinstian A: Prevalence of chronic kidney disease: A systematic review and metaanalysis. Clinical Nephrology 2009, 71:244-254.

21. Glas AS, Lijmer JG, Prins MH, Bonsel GJ, Bossuyt PM: The diagnostic odds ratio: a single indicator of test performance. J Clin Epidemiol 2003, 56(11):1129-1135.

22. Youden WJ: Index for rating diagnostic tests. Cancer 1950, 3(1):32-35.

23. Guyatt G, Rennie D, Meade MO, Cook DJ: Users guides to the medical literature: Essentials of evidence-based clinical practice. New York: McGraw Hill; 2008.

\section{Pre-publication history}

The pre-publication history for this paper can be accessed here: http://www.biomedcentral.com/1471-2369/12/45/prepub

doi:10.1186/1471-2369-12-45

Cite this article as: Thakkinstian et al:: A simplified clinical prediction score of chronic kidney disease: A cross-sectional-survey study. BMC Nephrology 2011 12:45.

\section{Submit your next manuscript to BioMed Central and take full advantage of:}

- Convenient online submission

- Thorough peer review

- No space constraints or color figure charges

- Immediate publication on acceptance

- Inclusion in PubMed, CAS, Scopus and Google Scholar

- Research which is freely available for redistribution 